\title{
A new model to investigate whether regional crop rotation strategies can protect crops from fungal pathogens
}

\author{
$\underline{\text { R. Crete }}^{\text {a }}$ and M. Renton ${ }^{a}$ \\ ${ }^{a}$ School of Plant Biology and UWA Institute of Agriculture, The University of Western Australia, \\ 35 Stirling Hwy, Crawley, WA 6009 Australia \\ Email: remi.crete@uwa.edu.au
}

\begin{abstract}
Plant pathogenic fungi cause severe damage, widespread losses, and are challenging to manage. Control strategies rely on fungicides, deep tillage of the crop residues, use of resistant cultivars, and crop management (specific sowing period, crop rotations). Farmers must yearly allocate fields to different crops and choose among these crop management options. Far from being obvious, these decisions are critical because they modify farm productivity and profitability in the short and long run. We built a new model specifically to address the issues described above. The first version of the model describes the population dynamics of a fungal pathogen over a large agronomic region comprising a number of fields in which both susceptible and neutral (non-susceptible) crops are grown. We compared different rates of rotations to see what rotation strategies were optimal in maximising non-infected susceptible crop area and minimising infected crop area. Then we adapted the model to investigate the case where three different crops can be used in the landscape, one non-susceptible crop, one susceptible host crop with low resistance and one susceptible host crop with high resistance. Our results showed that for a wide majority of cases, the configuration where we rotated infested fields into neutral fields at a faster rate than we rotated neutral fields back into susceptible crop gave better yield overall by reducing fungal incidence.
\end{abstract}

Keywords: Durability, resistance breakdown, crop rotation 


\section{INTRODUCTION}

Plant pathogenic fungi cause severe damage, widespread losses, and are challenging to manage. These difficulties mainly arise from complex interactions between the crop, the pathogen, and climate variables. Some long standing historical examples include apple scab, caused by the pathogen Venturia inaequalis (Aylor, 1998), tobacco blue mould caused by Peronospora tabacina (Aylor, 1999). Another very topical example is phoma stem canker (Blackleg), caused by Leptosphaeria maculans and/or L. biglobosa. Blackleg is one of the most damaging diseases of oilseed rape culture worldwide (West et al. 2000; Fitt et al. 2006). This disease is of major economic importance, with yield losses of between 5 and $20 \%$ of the production in some places (Aubertot et al., 2004), but up to $100 \%$ in exceptional situations (West et al., 2000). The disease is most severe in areas of intensive oilseed rape production because L.maculans/L.biglobosa ascospores in particular are released from oilseed rape stubble and spread extensively via wind, while rain splashed conidia are released both from infested stubbles and from lesions on plants to cause secondary spread. In Australia in particular, blackleg is a polycyclic disease, with mutiple cycles of spore production and infection across the year (Li et al., 2006; Li et al., 2007).

As epidemics reduce crop yield, strategies combining several tactics are deployed to control them. Management strategies rely on disease control methods such as like fungicides, deep tillage of the residue, use of resistant cultivars, and crop management such as sowing during a specific period or crop rotations (Fitt et al., 2006; West et al., 2000; Sivasithamparam et al., 2005).

Because of the large number of factors involved in the interactions between crop, pathogen and environment, the use of mechanistic models (Colbach, 2010), whether stochastic or deterministic, are invaluable tools for integrating key behaviours and assessing whether a given strategy of crop management is relevant or not before to be applied. A number of mechanistic models have been developed and tested for fungal pathogens as well as specific other pests, for example, citrus tristeza virus (Gibson, 1997), anthracnose (Diggle et al., 2002), yellow rust of wheat (Soubeyrand et al., 2007), forest gap generated by bark beetles species (Zhu et al., 2008), and apple scab (Crété et al., 2013).

Rotations between crops are often used to reduce yield loss as well as to keep the durability of resistance of some crops to pathogens (Dury et al., 2012). We introduce here a new model representing the following situation. A landscape is composed of fields of two types : host crop and non-host crop for the disease. The non-host kind of field is neutral in the sense that it cannot be infected or cannot infect other fields. The neutral crop yield is also less valuable than non-infected host crop. The main role of this kind of crop is to stop the development of the disease occuring in a field, by being sown in this field in the place of the contaminated crop. The host kind of field has two possible states : susceptible (meaning non-infested), and infested. First, we start by studying the case where only one kind of host crop will interact with a disease and neutral crop. We successively modify and compare the different rates of rotations to see if a given type of rotation is better than the others, in terms of increase of yield. Then we look at the case where two different host crop can be used in the landscape (two different cultivars with resistance genes to the disease for example), and investigate how rotation between a host field and another one more resistant crop can influence the dynamic of the disease.

\section{THE SIN MODEL}

The SIN model (Susceptible - Infested - Neutral) describes the population dynamics of a fungal pathogen over a large agronomic region comprising a number of fields in which both susceptible or neutral crops are grown (neutral meaning that the crop cannot be infected by the pathogen). The following equations describe the between-field (regional) dynamics:

$$
\begin{array}{cc}
\text { Susceptible fields : } & d S / d t=v I\left[t_{h}-(S+I)\right]-\lambda I S \\
\text { Infested fields : } \quad & d I / d t=\lambda I S-\mu I\left[T_{h}-(S+I)\right] \\
\text { Neutral fields : } \quad d N / d t=\mu I\left[T_{h}-(S+I)\right]-v I\left[t_{h}-(S+I)\right]
\end{array}
$$

The landscape is composed of a number $T_{h}$ of fields. We consider that the number of fields for the susceptible host is limited to a threshold value $t_{h}$, meaning that for all time, $S+I \leq t_{h}$. Then the number of neutral host field that are grown equals $T_{h}-(S+I)$. We note that the minimum number of neutral fields is $T_{h}-t_{h}$.

Infested fields produce spores, which escape the field, are blown away and initiate epidemics in susceptible fields at the rate $\lambda$. We will assume that each infested field contains an epidemic of equal size and produces the same quantity of spores. Epidemics within fields become extinct at rate $\mu$ due to crop rotation with a neutral field. Then the neutral fields are replaced by susceptible fields at the rate $v$. 
R. Crete and M. Renton, A new model to investigate whether ...

We note that the evolution of neutral fields quantity may be rewritten in the following way:

$$
d N / d t=I\left[\mu T_{h}-v t_{h}+(v-\mu)(S+I)\right]
$$

We will now study the evolution of the system of fields, depending on the growth of the disease $(\lambda)$, both rates of rotation $(I \rightarrow N$ and $N \rightarrow S$ ), and on relevant initial conditions. The latest relation allows us to see the following facts:

In a very simple scenario, we can assume that $T_{h}=t_{h}$. That means that in our model, no neutral field are possible, and all fields are the susceptible crop. We note that $S+I=T_{h}$ so it is constant, and the model reduces to a logistic model :

$$
\begin{array}{lrl}
\text { Susceptible fields : } & d S / d t=-\lambda I S \\
\text { Infested fields : } & d I / d t=\lambda I S
\end{array}
$$

So in the following we will always assume that $T_{h}>t_{h}$. We will fix the threshold $T_{h}$ at the value 10000 , the threshold for the susceptible crop will be equal to 9000, meaning that 1000 neutral fields we be grown at the beginning. We will study the cases where $v=\mu, \mu>v$ and $\mu<v$, and how the dynamics in each case depends on different epidemic spread rates (different $\lambda$ ).

When $\mu=v$, we can write $d N / d t=\mu I\left[T_{h}-t_{h}\right]$, meaning that the quantity of neutral field grows until there is no infested field anymore. Furthermore, when $I=0$, we have $d S / d t=d I / d t=d N / d t=0$, and so the system dynamics reaches a steady state. Figure 1 represents such a case of evolution for different disease intensities. When the speed of the disease is very low compared with the rate of rotation, infested field vanishes quickly, replaced by neutral fields, themselves replaced by susceptible ones. At the end we only have disease free susceptible fields and neutral fields. For the subfigure in the middle, the speed of the disease at the beginning massively reduces the number of susceptible fields. Even if the rotation gives some susceptible field back in time, at the end (steady state of the model) there are more neutral fields than susceptible fields. As we assume that the culture of susceptible field is more valuable in terms of return on investment that the neutral fields, this rotation strategy is not attractive. Then the last display shows what happens with an even higher spread rate; at the end there is only a small percentage of susceptible fields left.
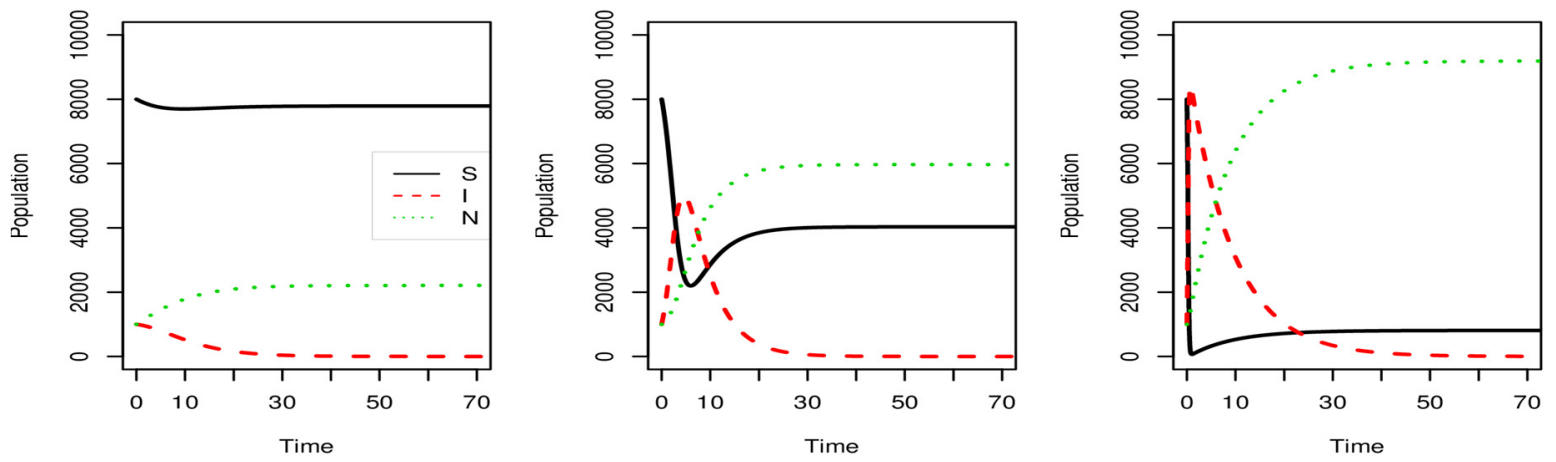

Figure 1. Representation of the evolution of the SIN system when 1000 fields are infested in a region of 10000 fields, whose number of neutral fields equals $1000 . \mu=v=0.0001, \lambda$ successively equals to $0.00001,0.0001$ and 0.001 from right to left.

When $\mu>v$, that means that we rotate infested fields into neutral fields at a faster rate than we rotate neutral fields back into susceptible crop. Some example realisations of simulations of three different scenarios are shown in Figure 2. The first subfigure (on the left) only differs from the first subfigure of Figure 1 through the value of $\mu$ which is ten times bigger. This ensures that there is almost no loss in terms of susceptible fields, with almost all the infested fields being absorbed by the neutral fields. With the graph in the middle we can note that there is no overall return of neutral fields towards susceptible fields, meaning the number of susceptible remains constant after a period of decrease. However, the final level of susceptible fields is higher than the corresponding level of the Figure 1. 
R. Crete and M. Renton, A new model to investigate whether ...
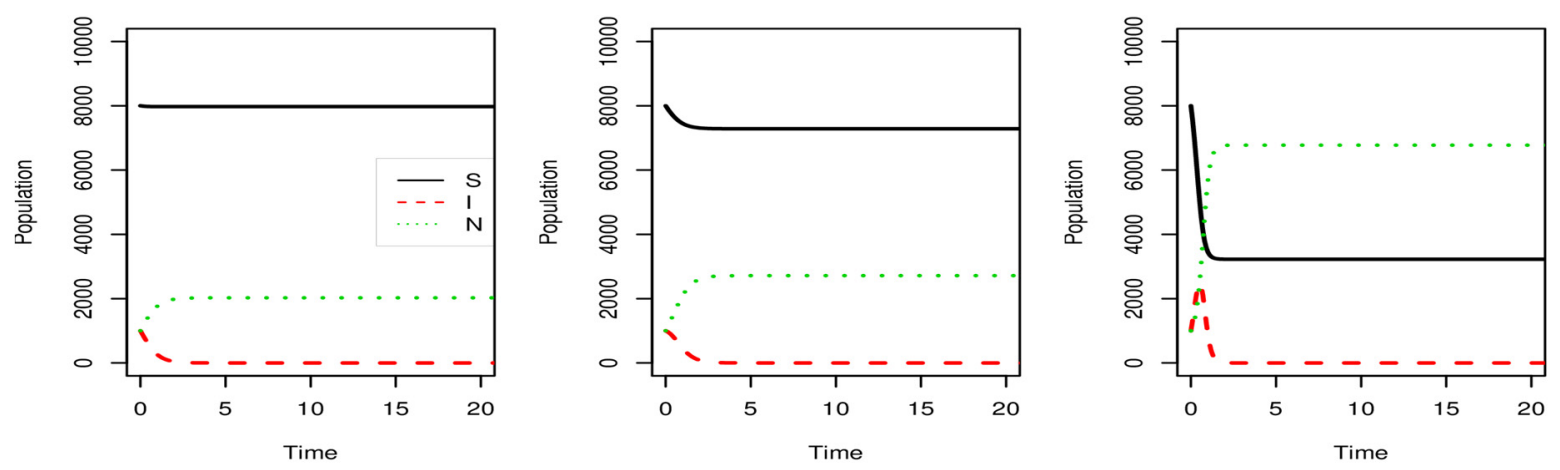

Figure 2. Representation of the evolution of the SIN system when 1000 fields are infested in a region of 10000 fields, whose number of neutral fields equals $1000 . \mu=0.001$ and $v=0.0001, \lambda$ successively equals to 0.00001 , 0.0001 and 0.0005 from right to left.

When $\mu<v$, we rotate neutral fields into susceptible fields more often than we rotate infested fields to neutral fields. Figure 3 represents a case where $d N / d t \approx 0.1$ so that the growth of $N$ is really limited. That is why the level of $N$ looks approximately constant for the three subfigures. In the case of the first subfigure, compared to the first subfigure in the first and the second figures, we note that the infested fields are seemingly essentially turned into susceptible fields. On the other hand, for the two other subfigures, farmers following these strategies will have important yield losses (big quantity of infested field at the steady state). In order to allow neutral fields to increase in number, we want to increase the value of the derivative $d N / d t$. To achieve that, we define $\mu$ in function of $v, t_{h}$ and $T_{h}$ in the following way: $\mu=v * t_{h} / T_{h}$. The behaviour of the model under these conditions can be seen in Figure 4 and is close to the behaviour of the case $\mu=v$, with the behaviour of some increase in the proportion of susceptible fields over a certain time period.
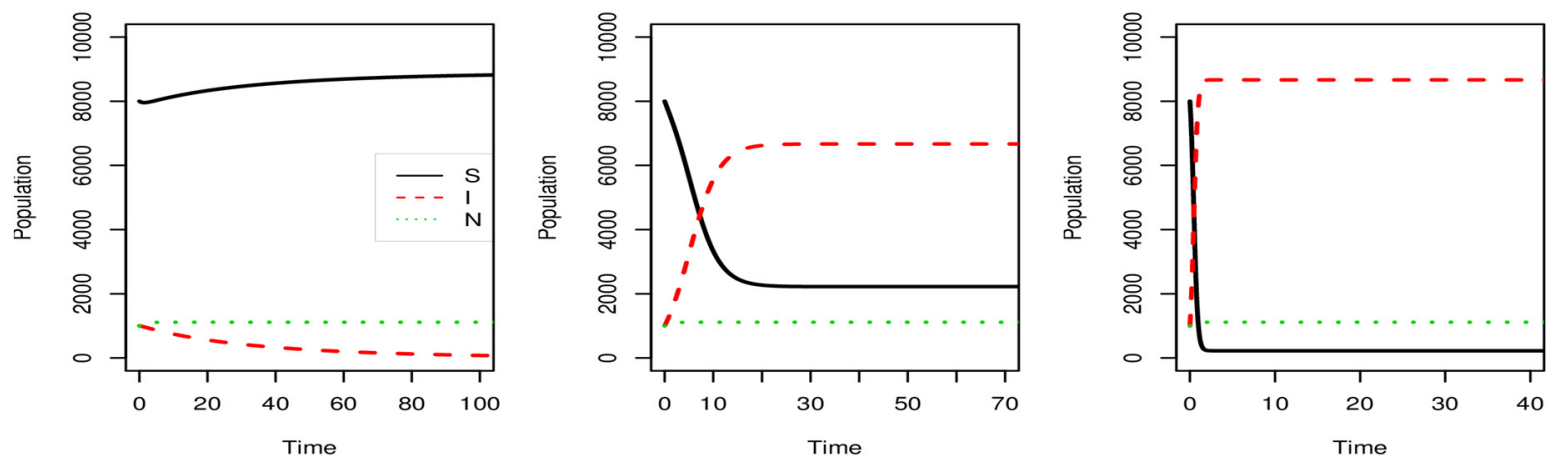

Figure 3. Representation of the evolution of the SIN system when 1000 fields are infested in a region of 10000 fields, whose number of neutral fields equals $1000 . \mu=0.0001$ and $v=0.001, \lambda$ successively equals to 0.00001 , 0.00005 and 0.0005 from right to left. 

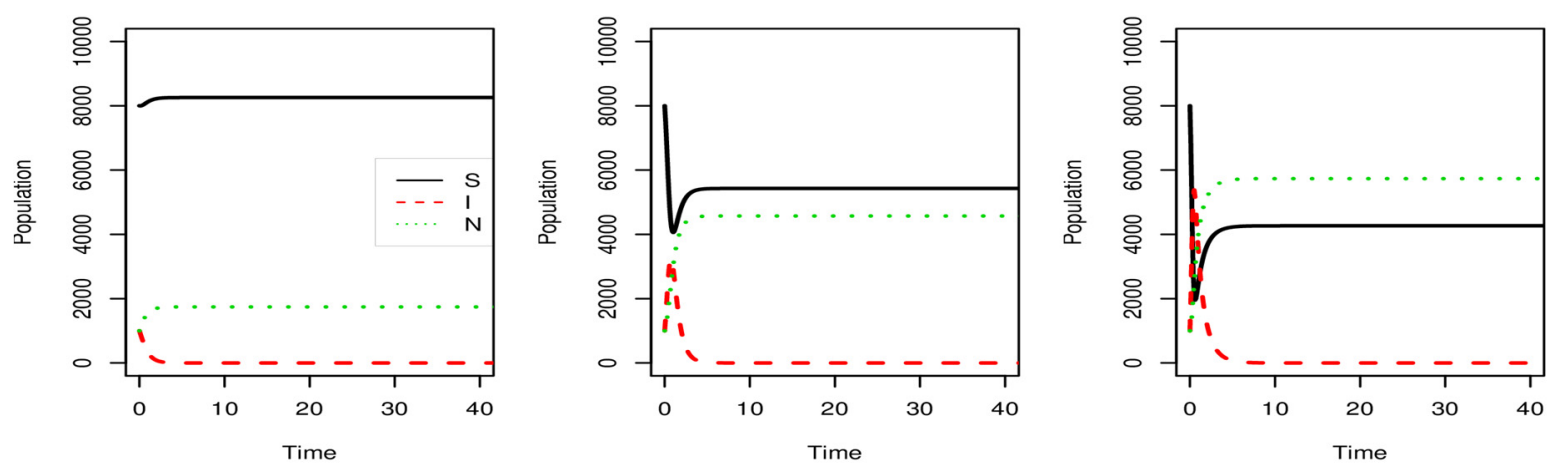

Figure 4. Representation of the evolution of the SIN system when 1000 fields are infested in a region of 10000 fields, whose number of neutral fields equals 1000. $\mu=0.0009$ and $v=0.001$, when $\mu=v^{*} t_{h} / T_{h}, \lambda$ successively equals to $0.00001,0.0005$ and 0.001 from right to left.

\section{APPLICATION OF THE SIN MODEL}

We adapt the SIN model to the case where the host crops are divided into two categories: (10) and (11). These numbers can be interpreted as the genotypes of the two cultivars grown, with a system of two resistance genes, the number 0 meaning the absence of the allele conferring resistance, while the value 1 indicates its presence. Thus we can consider that the cultivar (11) has one more gene of resistance than the cultivar (10). We allow an additional kind of rotation, which is that (10)-infested field may be rotated into (11)-susceptible field $(\omega)$. For each cultivar there is a pathogen strain virulence genes that can infect them, with $\lambda_{10}=0.0005$ and $\lambda_{11}=0.00025$ representing the infective pressure of these strains. The fact that $\lambda_{11}$ is lower than $\lambda_{10}$ can be interpreted like a fitness penalty due to an extra gene of resistance for (11)-host crop. The equations governing behaviour in this application are thus:

$$
\begin{aligned}
& d S_{10} / d t=v I_{10}\left[t_{h 10}-\left(S_{10}+I_{10}\right)\right]-\lambda_{10} I_{10} S_{10} \\
& d S_{11} / d t=v I_{11}\left[t_{h 11}-\left(S_{11}+I_{11}\right)\right]-\lambda_{11} I_{11} S_{11}+\omega I_{10}\left[t_{h 11}-\left(S_{11}+I_{11}\right)\right] \\
& d I_{10} / d t=\lambda_{10} I_{10} S_{10}-\mu I_{10} N-\omega I_{10}\left[t_{h 11}-\left(S_{11}+I_{11}\right)\right] \\
& d I_{11} / d t=\lambda_{11} I_{11} S_{11}-\mu I_{11} N \\
& d N / d t=\mu\left(I_{10}+I_{11}\right) N-v I_{10}\left[t_{h 10}-\left(S_{10}+I_{10}\right)\right]-v I_{11}\left[t_{h 11}-\left(S_{11}+I_{11}\right)\right]
\end{aligned}
$$

We assume the limit number of fields sown with these cultivars is respectively $t_{h 11}$ and $t_{h 10}$ with their values are fixed at 4500. The total number of fields is still 10000, and the quantity of neutral fields is 1000 . At the beginning of the example outputs shown in Figures 5 and $6, I_{10}=I_{11}=100$ fields. We can see for both of these figures that the choice of $\mu>v$ looks to be better than the other rotation strategies.
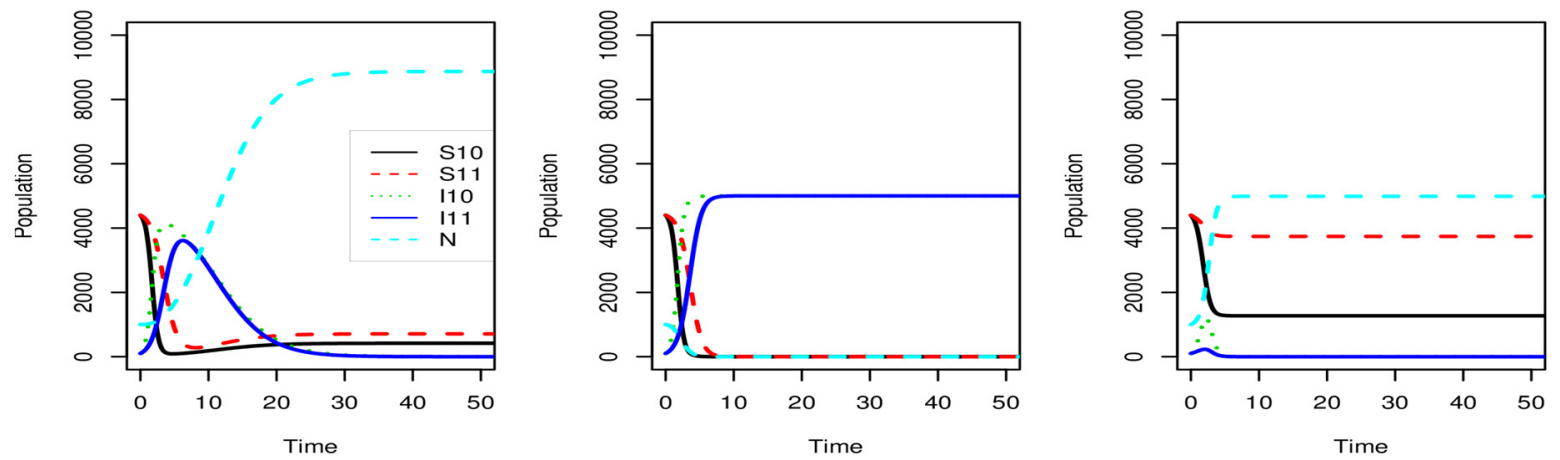

Figure 5. Representation of the evolution of the SIN system applied to two different host crop, when $\omega=0$. From left to right : $\mu=v=0.00005 ; \mu=0.00005$ and $v=0.0005 ; \mu=0.0005$ and $v=0.00005$ 

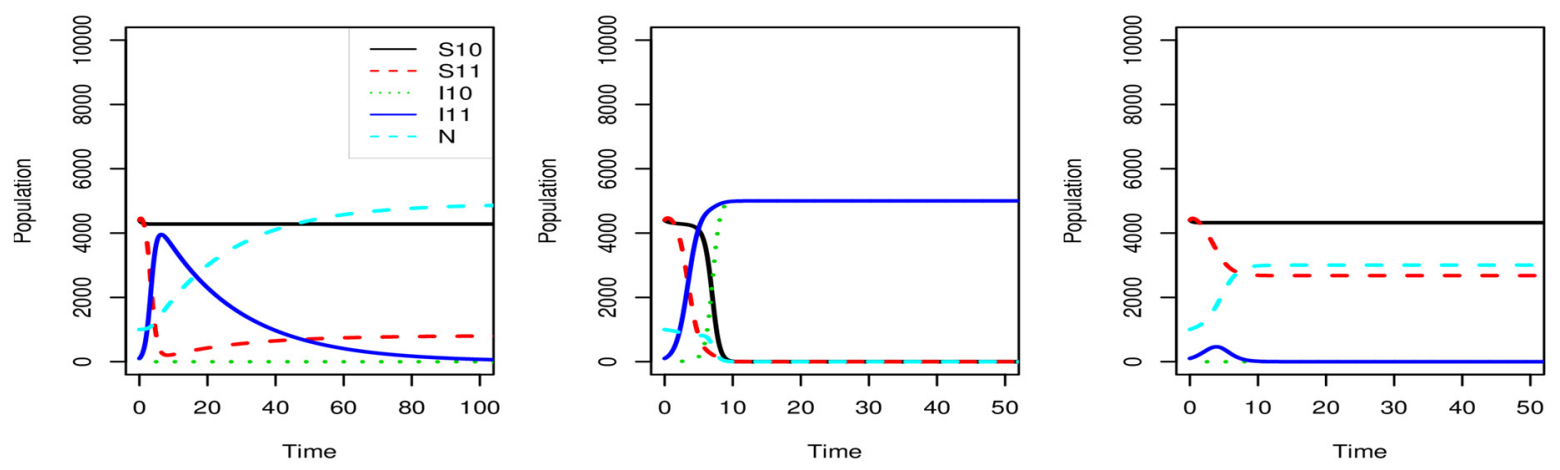

Figure 6. Representation of the evolution of the SIN system applied to two different host crop, when $\omega=0.01$. From left to right : $\mu=v=0.00005 ; \mu=0.00005$ and $v=0.0005 ; \mu=0.0005$ and $v=0.00005$

\section{CONCLUSIONS}

This paper proposed a new model of crop rotation based on the use of neutral crops, which are protected from fungal disease that may develop within a susceptible crop. This model is based on rates of rotation, meaning the frequencies at which a farmer will replace a given kind of crop by another one. We illustrated the behaviour of this ordinary differential equation system, through different rate of rotation ( $\mu$ and $v)$, with increasing levels of disease spread (reflected in increasing $\lambda$ ). It results that the rotation strategy where $\mu>v$ looks the best, with the exception of the case where the disease dynamics is very low, in which case $\mu<v$ looks to be the best. We found similar results in a more complicated case, when the host crop was separated into two different genotypes, and the disease is assumed to have two different corresponding strains. This kind of model (Van den Bosch and Gilligan, 2003, Parnell et al. 2006) may be hard to understand by farmers, because of its time structure. Here the time is continuous and the parameters are fixed (constant) with time. It could be tempting to increase $v$ for the scenarios where infected fields get low (toward zero), once this low value is reached. This model will be more useful when migration and mutation parameters are taken into account.

\section{ACKNOWLEDGEMENT}

The authors acknowledge support from the GRDC that helped fund this research.

\section{REFERENCES}

Aubertot, J., Pinochet, X., and Doré T. (2004). The effects of sowing date and nitrogen availability during vegetative stages on leptosphaeria maculans development on winter oilseed rape. Crop Protection, 23, 635-645.

Aylor, D. (1998). Aerobiology of apple scab. Plant Disease, 82, 838-849.

Aylor, D. (1999). Biophysical scaling and the passive dispersal of fungus spores: relationship to integrated pest management. Agricultural and Forest Meteorology, 97, 275-292.

Colbach, N. (2010). Modelling cropping system effects on crop pest dynamics: How to compromise between process analysis and decision aid. Plant Science, 179, 1-13.

Crété, R., Pumo, B., Soubeyrand, S., Didelot, F., and Caffier, V. (2013). A continuous time-and-state epidemic model fitted to ordinal categorical data observed on a lattice at discrete times. Journal of Agricultural, Biological, and Environmental Statistics, 18(4), 538-555.

Diggle, A., Salam, M., Thomas, G., Yang, H., O’Connell, M., and Sweetingham, M. (2002). Anthracnosetracer: A spatiotemporal model for simulating the spread of anthracnose in a lupin field. Phytopathology, 92, 1110-1121.

Dury, J., Schaller, N., Garcia, F., Reynaud, A., and Bergez, J. (2012). Models to support cropping plan and crop rotation decisions. Agronomy for Sustainable Development, 32, 567-580. 
R. Crete and M. Renton, A new model to investigate whether ...

Fitt, B., Brun, H., Barbetti, M., and Rimmer, S. (2006). Worldwide importance of phoma stem canker (leptosphaeria maculans and 1. biglobosa) on oilseed rape (brassica napus). European Journal of Plant Pathology, 114, 3-15.

Gibson, G. (1997). Markov chain monte carlo methods for fitting spatiotemporal stochastic models in plant epidemiology. Applied Statistics, 46, 215-233.

Li, H., Sivasithamparam, K., and Barbetti, M. (2006). Evidence supporting the polycyclic nature of blackleg disease (leptosphaeria maculans) of oilseed rape in australia and implications for disease management. Brassica, 8, 65-69.

Li, H., Sivasithamparam, K., and Barbetti, M. (2007). Blackleg disease (leptosphaeria maculans) on oilseed rape - evidence for it being a polycyclic disease in Australia and implications for disease management. Proceedings of the 10th International Rapeseed Congress, Wuhan, China, March 2007. Science Press USA Inc., Monmouth Junction, NJ, USA. I, 416-419.

Parnell, S., Van den Bosch, F., and Gilligan, C. (2006). Large-scale fungicide spray heterogeneity and the regional spread of resistant pathogen strains. Phytopathology (96), 549-555.

Sivasithamparam, K., Barbetti, M., and Li, H. (2005). Recurring challenges from a necrotrophic fungal plant pathogen: a case study with leptosphaeria maculans (causal agent of blackleg disease in brassicas) in Western Australia. Annals of Botany, 96, 363-377.

Soubeyrand, S., Enjalbert, J., Sanchez, A., and Sache, I. (2007). Anisotropy, in density and in distance, of the dispersal of yellow rust of wheat: Experiments in large field plots and estimation. Phytopathology, 97(10), $1315-1324$.

Van den Bosch, F. and Gilligan, C. (2003). Measures of durability of resistance. Phytopathology, 93, 616-625.

West, J., Kharbanda, P., Barbetti, M., and Fitt, B. (2000). Epidemiology and management of leptosphaeria maculans (phoma stem canker) on oilseed rape in australia, canada and europe. Plant Pathology, 50, 10-27.

Zhu, J., Rasmussen, J., Moller, J., Aukema, B., and Raffa, K. (2008). Spatial-temporal modeling of forest gaps generated by colonization from below- and above-ground bark beetle species. Journal of the American Statistical Association, 103(481), 162-177. 